\title{
ALTERNATIVAS PARA DISCIPLINAR O EFEITO DE DISTORÇÃO DO PREÇO E DO COMÉRCIO MUNDIAL CAUSADO PELOS SUBSÍDIOS AGRÍCOLAS
}

\author{
Cinthia Cabral da Costa* \\ André Meloni Nassar ${ }^{\dagger}$ \\ MARCos SAWAYA JANK ${ }^{\ddagger}$
}

\begin{abstract}
Resumo
Os limites dos subsídios agrícolas consolidados na Rodada Uruguai podem causar altos níveis de distorção no mercado dos produtos subsidiados. O presente estudo propõe limites nos valores de subsídios, por produto, calculados de maneira a se ter um nível máximo de distorção de preços no mercado mundial. Foi estimado um modelo de equilíbrio parcial para cada produto. Os resultados, para os principais produtos subsidiados nos EUA, tiveram como valor de subsídio máximo para distorcer um limite de $2 \%$ do seu preço internacional: US $\$ 1,0$ bilhão para soja; US\$ 0,6 bilhão para o algodão; US\$ 0,2 bilhão para arroz; US\$ 1,4 bilhão para o milho e US\$ 1,3 bilhão para o trigo. Portanto, sugere-se que estes valores sejam considerados como parâmetro para os tetos de subsídio por produto.
\end{abstract}

Palavras-chave: subsídios agrícolas; distorção no preço; comércio internacional.

\begin{abstract}
The limits to agricultural subsidies established in the Uruguay Round Agreement on Agriculture could cause trade-distorting domestic support. This study suggests limits in the subsidy value to level the maximum price distortion for each product. A partial equilibrium model was estimated for the products. The maximum value of subsidy to main agricultural products in the country-regionplaceUSA., causing a two percentage limit in their world price, were: US\$ 1.0 billion to soybean; US\$ 0.6 billion to cotton; US\$ 0.2 billion to rice; US\$ 1.4 to corn and US\$ 1.3 billion to wheat. Therefore, those patterns of product value could be used as a parameter to product subsidy cap.
\end{abstract}

Palavras-chave: agricultural subsidies, price distortion, international trade. JEL classification: F13

\footnotetext{
* Professora da Universidade Federal de São Carlos, Campus de Sorocaba. Endereço: Rodovia João Leme dos Santos, km 110 - SP-264. Bairro Itinga. CEP: 18052-780. Sorocaba, SP. E-mail: cinthia-costa@ufscar.br.

${ }^{\dagger}$ Diretor Geral do Instituto de Estudos do Comércio e Negociações Internacionais (ICONE). Email: amnassar@iconebrasil.org.br

‡ Presidente da UNICA e Professor da Faculdade de Economia, Administração e Contabilidade da Universidade de São Paulo (FEA-USP). E-mail: msjank@unica.com.br.
} 


\section{Introdução}

Conforme descrito por Jales e Nassar (2005), os países desenvolvidos, principalmente a União Européia (UE), os Estados Unidos e o Japão, são os responsáveis pela maior parte dos subsídios agrícolas mundiais e os principais responsáveis pelas distorções causadas no mercado mundial. A possibilidade de uso destes subsídios foi concedida na Rodada do Uruguai e na Rodada de Doha, em que um esforço para disciplinar estes subsídios é foi um dos objetivos centrais das negociações. Entretanto, nesse mesmo estudo, os autores descrevem porque são insuficientes os cortes nos subsídios domésticos ${ }^{1}$ propostos pelos EUA e pela UE na Rodada de Doha. Assim, uma das alternativas descritas pelos autores para cortes efetivos nos atuais níveis de subsídios domésticos é estabelecer disciplinas específicas por produto. Nesse sentido, este o presente estudo procurou estabelecer, com base na teoria econômica e no Acordo Sobre Subsídios e Medidas Compensatórias, os limites, por produto, a serem definidos como disciplinas para os subsídios americanos, nos principais produtos subsidiados nos EUA. A justificativa para se escolher os EUA como estudo de caso é porque esse país é o que mais resiste à redução dos atuais níveis de subsídio.

A teoria econômica indica que, ao subsidiar a produção de determinado bem, o país pode provocar distorções não só no mercado doméstico, mas também no mercado mundial. No mercado doméstico, o subsídio impacta positivamente a produção. Ao aumentar a oferta do produto subsidiado, sem alteração correspondente na demanda, o preço do mesmo tende a cair. Essa redução no preço do produto subsidiado pode se estender para o mercado mundial caso o excesso de produção decorrente do subsídio seja significante nesse mercado. Entretanto, tal efeito é claramente condenado pelo parágrafo terceiro do Artigo 6 do Acordo Sobre Subsídios e Medidas Compensatórias, que diz:

“3. Ocorrerá grave dano no sentido do parágrafo (c) do Artigo 5 sempre que ocorra um ou a combinação de vários dos seguintes efeitos:

1. deslocar ou impedir a importação de produto similar produzido por outro Membro no mercado do Membro outorgante do subsídio;

2. deslocar ou impedir a exportação de produto similar produzido por um Membro no mercado de terceiro país;

3. provocar significativa redução do preço do produto subsidiado em relação ao preço do produto similar de outro Membro no mesmo mercado, ou significativa contenção de aumento de preços, redução de preços ou perda de vendas no mesmo mercado;

4. aumentar a participação no mercado mundial de determinado produto primário ou de base subsidiado pelo Membro outorgante, quando se compara com a participação média que o Membro detinha no período de três anos anteriores e quando tal aumento se mantém como firme tendência durante algum tempo após a concessão dos subsídios."

\footnotetext{
${ }^{1}$ Para maiores detalhes sobre as propostas e análise das mesmas, ver em Jales e Nassar (2005).
} 
Assim, o efeito depressivo no preço ao nível do mercado mundial é um indicativo do efeito prejudicial do subsídio. Esse impacto tem sido um indicativo econômico para julgamento contra subsídios na Organização Mundial do Comércio (OMC), como no caso do painel do algodão, por exemplo.

$\mathrm{Na}$ atual Rodada de Negociações da OMC (Rodada de Doha), a redução dos subsídios domésticos agrícolas, juntamente com a questão do acesso a mercados, está entre os temas mais importantes. Portanto, este trabalho tem como objetivos: (i) estimar, economicamente, os níveis de subsídios máximos que poderiam ser aplicado nos principais produtos subsidiados pelos EUA, de maneira a causar menores níveis de dano no preço mundial, e, (ii) com base nos resultados obtidos, contribuir com uma proposta para disciplinar os atuais níveis de apoio doméstico, por produto.

\section{Revisão da Literatura}

Os trabalhos revisados que tratam do tema de impactos dos subsídios agrícolas foram realizados analisando os resultados em termos de preço mundial, produção e comércio. Estes dois últimos, avaliando os resultados no país que subsidia e no resto do mundo. Nestes trabalhos foram utilizados modelos de equilíbrio parcial para cada um dos produtos analisados (Sumner 2003, Poonyth et al. 2004, Sumner 2005, Brandão e Lima 2006).

Esta revisão foi importante para este trabalho na medida em que os modelos utilizados nestes trabalhos estão calcados nas equações de oferta e demanda de tais mercados, da mesma maneira que como foi realizado no presente estudo. A principal diferença deste trabalho é que, com base nos resultados de impactos do subsídio sobre o preço, foi estabelecido um valor máximo de subsídio que o país pode conceder para que este impacto esteja num determinado patamar, aqui definido como razoável pelos autores.

Sendo a base metodológica muito semelhante, a Tabela 1 mostra uma comparação dos parâmetros utilizados nos quatro trabalhos revistos para a formação das equações de oferta e de demanda nos mercados analisados em cada um.

Portanto, verifica-se uma grande similaridade no modelo teórico dos trabalhos descritos na Tabela 1. Basicamente, a demanda é influenciada pelo preço doméstico do produto analisado e a oferta é uma função, não apenas deste preço, como também da rentabilidade do produtor. Tal rentabilidade está associada ao nível de subsídio recebido. As equações de oferta e de demanda utilizadas neste trabalho seguiram estas mesmas relações teóricas, tendo uma proximidade maior com o modelo descrito por Sumner (2003, 2005).

\section{Metodologia}

Para estimar o impacto do subsídio doméstico de um país no nível do preço mundial, parte-se de um modelo econômico de oferta e demanda mundial para o produto agrícola que recebe subsídio. Portanto, será adotado um modelo de equilíbrio parcial, considerando o mercado mundial para cada uma das commodities analisadas: soja, arroz, algodão, milho e trigo. O modelo teórico descrito a seguir é o mesmo para os cinco produtos analisados. 
Tabela 1: Comparação entre os parâmetros utilizados nas equações de oferta e de demanda nos trabalhos revisados

\begin{tabular}{lll}
\hline & Equação de demanda & Equação de oferta \\
\hline Sumner, 2003 & Preço do produto & $\begin{array}{l}\text { Preço do produto } \\
\text { Rentabilidade do } \\
\text { produto }\end{array}$ \\
Poonyth et al., 2004 & $\begin{array}{l}\text { Preço doméstico do } \\
\text { produto } \\
\text { Preço do produto } \\
\text { substituto }\end{array}$ & $\begin{array}{l}\text { Preço doméstico do } \\
\text { produto } \\
\text { Preço do produto } \\
\text { substituto }\end{array}$ \\
& Preço do produto & $\begin{array}{l}\text { Preço do produto } \\
\text { Subsídio unitário } \\
\text { Sumner, 2005 }\end{array}$ \\
& & $\begin{array}{l}\text { Grau de confiança no } \\
\text { recebimento do } \\
\text { subsídio }\end{array}$ \\
& & $\begin{array}{l}\text { Rentabilidade do } \\
\text { produto }\end{array}$ \\
Brandão e Lima, 2006 do produto & Preçada do \\
& & Produtividade \\
\hline
\end{tabular}

O modelo de equilíbrio parcial é mais efetivo para este tipo de análise do que o modelo de equilíbrio geral, uma vez que os modelos computáveis de equilíbrio geral disponíveis não detalham como setores da economia os produtos que são objetos deste estudo. Mesmo em trabalhos que realizaram este detalhamento prévio no modelo, a grande abrangência necessária das informações neste tipo de metodologia pode impedir a riqueza de detalhamento dos resultados necessária para a análise proposta neste trabalho. $\mathrm{O}$ modelo computável de equilíbrio geral possui também uma importância na quantidade de informações que disponibiliza como resultado de um choque exógeno no sistema, mas o faz em detrimento, muitas vezes, da confiabilidade de algumas informações específicas. Neste trabalho há três características que justificam o uso do modelo de equilíbrio parcial como mais apropriado ao de equilíbrio geral. São elas: (i) especificidade dos mercados a serem analisados; (ii) especificidade do resultado a ser obtido - impacto na redução do preço mundial de algumas commodities, estabelecendo um limite para o nível máximo de subsídios que poderiam ser dados às mesmas; e (iii) alta confiabilidade no resultado a ser obtido, uma vez que poderão ser utilizados para negociações específicas para este problema.

Utilizando os Estados Unidos como exemplo de país que subsidia um determinado produto $i$, tem-se que:

$$
\begin{aligned}
& d \ln S_{u}=\varepsilon_{u}\left(d \ln R_{u}\right) \\
& d \ln D_{u}=\eta_{u}(d \ln P)
\end{aligned}
$$

que indica, respectivamente, a derivada $(d)$ do logaritmo $(\ln )$ das equações de oferta e demanda do produto $i$ nos EUA. A variável $S_{u}$ representa a oferta americana; $\varepsilon_{u}$ é a elasticidade preço de oferta doméstica; $R_{u}$ é o valor total 
recebido pelo produtor nos EUA; $D_{u}$ é a demanda doméstica e; $\eta_{u}$ é a elasticidade preço da demanda doméstica. As equações de oferta e de demanda são escritas na forma de derivada do logaritmo para que os coeficientes sejam lidos, diretamente, como o valor da elasticidade correspondente.

O valor de $R_{u}$ pode ser composto pelo preço recebido pelo produtor $(P)$ mais o tamanho do subsídio $(g)$ que afeta diretamente sua produção $\left(R_{u}=\right.$ $P+g)$. Para estimar o valor do subsídio que afeta diretamente a produção, o valor estimado do subsídio total unitário (subsídio dividido pelo volume produzido) $-g^{\prime}$ - deve ser multiplicado por um coeficiente que representa a confiança do produtor americano em relação àquela receita $(\gamma) \cdot{ }^{2} \mathrm{O}$ coeficiente $\gamma$ também pode ser definido como o grau de incentivo da receita do governo (subsídio) na produção relativo à receita do mercado (preço mundial), assim: $g=\gamma \times g^{\prime}$. Nesse caso, uma parte de $R_{u}$ é representada por $P$ e outra parte é representado por $g$. Assim, $d \ln R_{u}=\alpha d \ln P+(1-\alpha) d \ln g$, em que:

$$
\begin{gathered}
\alpha=\frac{P}{R_{u}}=\frac{P}{P+g}=\frac{P}{P+\gamma g^{\prime}} \frac{1 / g^{\prime}}{1 / g^{\prime}}=\frac{P / g^{\prime}}{P / g^{\prime}+\gamma} \\
(1-\alpha)=\frac{g}{R_{u}}=\frac{g}{P+g}=\frac{\gamma g^{\prime}}{P+\gamma g^{\prime}} \frac{1 / g^{\prime}}{1 / g^{\prime}}=\frac{\gamma}{P / g^{\prime}+\gamma}
\end{gathered}
$$

As derivadas do logaritmo das equações de oferta e demanda do resto do mundo para o produto $i$ são:

$$
\begin{aligned}
& d \ln S_{r}=\varepsilon_{r}(d \ln P) \\
& d \ln D_{r}=\eta_{r}(d \ln P)
\end{aligned}
$$

em que o subscrito $r$ indica que a oferta, a demanda e as elasticidades correspondentes são para o resto do mundo, excetuando-se EUA.

A oferta mundial é a soma da oferta americana mais a do resto do mundo:

$$
d \ln S_{w}=\delta_{S U} d \ln S_{u}+\left(1-\delta_{S U}\right) d \ln S_{r}
$$

que fica:

$$
d \ln S_{w}=\delta_{S U} \varepsilon_{u} \alpha d \ln P+\delta_{S U} \varepsilon_{u}(1-\alpha) d \ln g+\left(1-\delta_{S U}\right) \varepsilon_{r} d \ln P
$$

e a demanda mundial é a soma das demandas americanas e do resto do mundo:

$$
d \ln D_{w}=\delta_{D U} \eta_{u} d \ln P+\left(1-\delta_{D U}\right) \eta_{r} d \ln P
$$

em que $\delta_{S U}$ e $\delta_{D U}$ são, respectivamente, a participação dos EUA na oferta e na demanda mundial do produto $i$. Portanto, $\left(1-\delta_{S U}\right)$ e $\left(1-\delta_{D U}\right)$ indicam a participação dos demais países na produção e no consumo mundial, respectivamente. Para o arroz (um dos produtos analisados neste trabalho) foi utilizada a participação dos EUA nas exportações e importações mundiais. Isso porque grande parte da produção mundial é realizada e consumida dentro da fazenda em áreas remotas de países da Ásia ou é protegida por quotas tarifárias de importação e não sujeita a forças de mercado.

\footnotetext{
${ }^{2}$ Este conceito foi proposto por Sumner (2005).
} 
Como no equilíbrio a oferta é igual à demanda, a equação base fica assim:

$$
\begin{array}{r}
\delta_{S U} \varepsilon_{u} \alpha d \ln P+\delta_{S U} \varepsilon_{u}(1-\alpha) d \ln g+\left(1-\delta_{S U}\right) \varepsilon_{r} d \ln P \\
=\delta_{D U} \eta_{u} d \ln P+\left(1-\delta_{D U}\right) \eta_{r} d \ln P
\end{array}
$$

Multiplicando ambos os lados da equação por $\frac{1}{d \ln g}$, e isolando $\alpha$, que é a variável que incorpora o tamanho do subsídio, temos:

$$
\begin{array}{r}
\delta_{S U} \varepsilon_{u} \alpha \frac{d \ln P}{d \ln g}+\delta_{S U} \varepsilon_{u} \frac{d \ln g}{d \ln g}-\delta_{S U} \varepsilon_{u} \alpha \frac{d \ln g}{d \ln g}+\left(1-\delta_{S U}\right) \varepsilon_{r} \frac{d \ln P}{d \ln g} \\
=\delta_{D U} \eta_{u} \frac{d \ln P}{d \ln g}+\left(1-\delta_{D U}\right) \eta_{r} \frac{d \ln P}{d \ln g} \\
\alpha=\frac{\delta_{D U} \eta_{u} \frac{d \ln P}{d \ln g}+\left(1-\delta_{D U}\right) \eta_{r} \frac{d \ln P}{d \ln g}-\left(1-\delta_{S U}\right) \varepsilon_{r} \frac{d \ln P}{d \ln g}-\delta_{S U} \varepsilon_{u}}{\delta_{S U} \varepsilon_{u}\left(\frac{d \ln P}{d \ln g}-1\right)}
\end{array}
$$

O impacto do subsídio no preço mundial é dado por $d \ln P / d \ln g$. Esse impacto pode então ser estabelecido $a$ priori, como sendo um limite em que o subsídio pode interferir no preço mundial. Assim, a equação (9) nos fornece um valor estimado de $\alpha$. Para calcular o percentual do subsídio no preço que gera o nível $\alpha$, como $\alpha=\frac{P / g^{\prime}}{P / g^{\prime}+\gamma}$, tem-se que então:

$$
P / g^{\prime}=\frac{\alpha \gamma}{1-\alpha}
$$

Finalmente, multiplicando o inverso da equação (10) por um preço de referência do produto e por um dado volume de produção de referência, tem-se o valor de subsídio total que o país pode conceder para o produto analisado:

$$
G=\frac{1}{P / g^{\prime}} \times P \times \text { quantidade produzida }
$$

em que o valor de $G$ seria então o subsídio estimado para um limite máximo de interferência no preço mundial.

Assim, o valor de $G$ estimado seria um guia para possíveis propostas de redução nos atuais níveis de subsídios. Esta metodologia foi aplicada para os principais produtos agrícolas subsidiados no mercado norte-americano: algodão, soja, milho, trigo e arroz.

Dos subsídios agrícolas totais norte-americanos, considerando o período de 1995-2000, segundo dados das notificações da OMC (WTO), tem-se que: $19 \%$ foram gastos no algodão (correspondendo a $29 \%$ do valor da produção dessa commodity), $18 \%$ foram para soja (o que pagou cerca de $11 \%$ do seu valor de produção), 37\% destinaram-se para produção de milho (o que significou $16 \%$ do valor da produção). O trigo utilizou 15\% do total (o que corresponde a $18 \%$ do valor da produção) e $4 \%$ dos subsídios aplicados naquele período foram para os produtores de arroz (significando $26 \%$ do valor da produção desse produto). Assim, como $93 \%$ dos subsídios agrícolas foram gastos com tais commodities, no período recente, este foi o universo dos mercados analisados. 


\section{Resultados e Discussão}

Inicialmente, foram descritos os valores das variáveis definidas nas equações (9) e (11), considerando as características do mercado agrícola americano, objeto deste estudo. Algumas variáveis foram calculadas, outras obtidas de fontes secundárias, e dados passados de preço e produção foram utilizados para estabelecer o valor de algumas variáveis. Nesse contexto, foi também apresentada uma breve discussão a respeito do nível de tolerância máximo para que o subsídio de um país possa impactar o nível de preço mundial. Os resultados dos níveis de subsídio máximo por produto e uma proposta de disciplina por produto, baseada nesse critério, foram finalmente obtidos.

\subsection{Parâmetros de oferta e demanda norte-americana e mundial}

Na equação (9) verifica-se a necessidade de se conhecer os valores de alguns parâmetros que indicam o comportamento dos agentes econômicos na oferta e na demanda nos mercados estudados. Alguns podem ser calculados, como a participação dos EUA na produção e no consumo mundial. Entretanto, há ainda outros parâmetros que devem ser estimados. São eles: a confiança do produtor em relação à receita do subsídio $(\gamma)$, a elasticidade preço de demanda dos consumidores dos EUA $\left(\eta_{u}\right)$, a elasticidade preço de demanda do resto do mundo $\left(\eta_{r}\right)$, a elasticidade preço de oferta dos produtores dos EUA $\left(\varepsilon_{u}\right)$ e a elasticidade preço de oferta dos produtores do resto do mundo $\left(\varepsilon_{r}\right)$.

Neste estudo, os parâmetros que devem ser estimados foram obtidos da literatura acadêmica e de instituições de pesquisa. A Tabela 2 descreve os valores dos coeficientes calculados e os obtidos de fontes secundárias de informação que foram utilizados neste estudo e as respectivas fontes destes dados. Os valores das elasticidades selecionados foram pesquisados de fontes diversas e, quando obtidas várias informações, selecionados com base na coerência e confiabilidade dos possíveis resultados encontrados.

Voltando à equação (9), observa-se que ainda falta definir o valor de $\frac{d \ln P}{d \ln g}$. Ou seja, o valor do percentual de depressão no preço mundial que seria aceitável de um país provocar pelo subsídio que aplica. Para isso, foi realizada uma análise descrita no próximo item. A definição do valor do impacto do subsídio sobre o preço mundial que um país pode ocasionar é um dos pontos mais importantes neste estudo e, também, depende da negociação entre os países. A seguir propomos um cenário provável de tolerância desse impacto, baseado em resultados de trabalhos de contestação dos níveis de alguns destes subsídios analisados.

\subsection{Limite máximo para impacto do subsídio no preço}

No Acordo Agrícola da Rodada Uruguai está definida a proibição de subsídio, na medida em que este cause uma depressão no nível de preço mundial. Entretanto, não há qualquer limite de tolerância indicado para essa interferência. A definição do nível de tolerância para a aplicação dos subsídios é crucial, uma vez que os atuais níveis de subsídios aplicados são elevados (mesmo considerando os níveis de corte que foram apresentadas nas últimas propostas das negociações da Rodada de Doha em dezembro de 2008). Os impactos no preço internacional dos atuais níveis de subsídios americanos de algumas commodities agrícolas foram descritos por Sumner (2005). Nesse estudo, Sum- 
Tabela 2: Valores dos coeficientes calculados e obtidos de estudos acadêmicos utilizados no cálculo da equação

\begin{tabular}{|c|c|c|}
\hline Parâmetro & Valor & Fonte \\
\hline \multicolumn{3}{|c|}{ Arroz } \\
\hline Gama & 0,6 & Sumner (2005) \\
\hline $\begin{array}{l}\text { Share da oferta (exportações americanas no } \\
\text { total mundial) }\end{array}$ & $12 \%$ & USDA (2007), média 1996/1997 a 2006/2007 \\
\hline $\begin{array}{l}\text { Share da demanda (importações americanas } \\
\text { no total mundial) }\end{array}$ & $2 \%$ & USDA (2007), média 1996/1997 a 2006/2008 \\
\hline Elasticidade preço da oferta dos EUA & 0,4 & FAPRI (2007) \\
\hline $\begin{array}{l}\text { Elasticidade preço da oferta no resto do } \\
\text { mundo }\end{array}$ & 0,21 & FAPRI (2007), média 21 países \\
\hline Elasticidade preço da demanda dos EUA & $-0,01$ & FAPRI (2007) \\
\hline $\begin{array}{l}\text { Elasticidade preço da demanda do resto do } \\
\text { mundo }\end{array}$ & $-0,17$ & FAPRI (2007), média 25 países \\
\hline \multicolumn{3}{|c|}{ Milho } \\
\hline Gama & 0,75 & Sumner (2005) \\
\hline Share da oferta & $40 \%$ & USDA (2007), média 1996/1997 a 2006/2007 \\
\hline Share da demanda & $32 \%$ & USDA (2007), média 1996/1997 a 2006/2008 \\
\hline Elasticidade preço da oferta dos EUA & 0,5 & JEFFERSON-MOORE, 2005. \\
\hline $\begin{array}{l}\text { Elasticidade preço da oferta no resto do } \\
\text { mundo }\end{array}$ & 0,23 & FAPRI (2007), média 25 países \\
\hline Elasticidade preço da demanda dos EUA & $-0,3$ & $\mathrm{USDA}^{a}$ \\
\hline $\begin{array}{l}\text { Elasticidade preço da demanda do resto do } \\
\text { mundo }\end{array}$ & $-0,2$ & FAPRI (2007), média 28países \\
\hline \multicolumn{3}{|c|}{ Algodão } \\
\hline Gama & 0,78 & Sumner (2005) \\
\hline Share da oferta & $19 \%$ & USDA (2007), média 1996/1997 a 2006/2007 \\
\hline Share da demanda & $9 \%$ & USDA (2007), média 1996/1997 a 2006/2008 \\
\hline Elasticidade preço da oferta dos EUA & 0,45 & BEACH et al., 2002. \\
\hline $\begin{array}{l}\text { Elasticidade preço da oferta no resto do } \\
\text { mundo }\end{array}$ & 0,18 & SHEPHERD, 2006. \\
\hline Elasticidade preço da demanda dos EUA & $-0,4$ & BEACH et al., 2002. \\
\hline $\begin{array}{l}\text { Elasticidade preço da demanda do resto do } \\
\text { mundo }\end{array}$ & $-0,26$ & POONYTH et al. 2004. \\
\hline \multicolumn{3}{|c|}{ Soja } \\
\hline Gama & 0,65 & \\
\hline $\begin{array}{l}\text { Share da oferta (exportações americanas no } \\
\text { total mundial) }\end{array}$ & $42 \%$ & USDA (2007), média 1996/1997 a 2006/2007 \\
\hline $\begin{array}{l}\text { Share da demanda (importações americanas } \\
\text { no total mundial) }\end{array}$ & $27 \%$ & USDA (2007), média 1996/1997 a 2006/2008 \\
\hline Elasticidade preço da oferta dos EUA & 0,27 & LIN et al., 2000. \\
\hline $\begin{array}{l}\text { Elasticidade preço da oferta no resto do } \\
\text { mundo }\end{array}$ & 0,33 & FAPRI (2007), média 11 países \\
\hline Elasticidade preço da demanda dos EUA & $-0,19$ & PIGGOTT, 2000. \\
\hline $\begin{array}{l}\text { Elasticidade preço da demanda do resto do } \\
\text { mundo }\end{array}$ & $-0,22$ & FAPRI (2007), média 11 países \\
\hline \multicolumn{3}{|c|}{ Trigo } \\
\hline Gama & 0,65 & Sumner (2005) \\
\hline Share da oferta & $26 \%$ & USDA (2007), média 1996/1997 a 2006/2007 \\
\hline Share da demanda & $2 \%$ & USDA (2007), média $1996 / 1997$ a $2006 / 2008$ \\
\hline Elasticidade preço da oferta dos EUA & 0,34 & LIN et al., 2000. \\
\hline $\begin{array}{l}\text { Elasticidade preço da oferta no resto do } \\
\text { mundo }\end{array}$ & 0,2 & FAPRI (2007), média 23 países \\
\hline Elasticidade preço da demanda dos EUA & $-0,1$ & FALCK-ZEPEDA et al., 2000. \\
\hline $\begin{array}{l}\text { Elasticidade preço da demanda do resto do } \\
\text { mundo }\end{array}$ & $-0,21$ & FAPRI (2007), média 25 países \\
\hline
\end{tabular}


ner mostrou que, considerando dados de 2003 a 2005, os subsídios americanos podem ter deprimido o preço mundial do milho em até $10 \%$, em $8 \%$ o preço do trigo e em $6 \%$ o preço do arroz. Em outro estudo do mesmo autor, que serviu de base para o caso do painel do algodão, identificou-se que os subsídios deprimiam o preço internacional desse produto em cerca de $10,8 \%$. Esse foi um dos resultados que provou o dano e contribuiu para o veredicto da $\mathrm{OMC}$, impondo ao governo norte-americano uma reforma daqueles níveis de subsídios.

Dados esses valores, um cenário possível de baixo nível de dano dos subsídios no preço mundial de commodities agrícolas seria um valor de, no máximo, $5 \%$ de impacto. Considerando ainda a característica das Rodadas de Negociações, cujo objetivo é a redução dos atuais níveis de proteção existentes, esse valor teria um nível de tolerância ainda menor do que 5\%. Neste estudo, ilustraremos a proposta para redução no nível de subsídios considerando alguns produtos agrícolas americanos, e supondo que a Rodada irá determinar um nível de tolerância aos subsídios domésticos que é de $2 \%$ de depressão no nível do preço mundial.

\subsection{Hipóteses de preço e quantidade produzida}

Finalmente, como demonstrado na equação (11), podemos ter vários níveis de subsídio para um mesmo nível máximo de interferência que o subsídio pode provocar no preço mundial em função de diferentes valores de preço e quantidade produzida. Pela equação (11) temos que, para um mesmo nível de produção, na situação de queda de preço, o nível do subsídio que impacta o preço mundial a certo nível estabelecido como crítico deveria ser menor do que em outra situação de preços normais. Essa constatação é importante no estabelecimento do nível crítico de subsídio $(G)$ que pode ser aplicado. Isso porque, no mundo real, os países aplicam maiores níveis de subsídio em situações de queda de preço. As Figuras 1 a 5 mostram essa situação nos produtos analisados neste estudo. Ou seja, se for definido um valor de $G$ com base em um determinado nível de preço e, no futuro, o preço for inferior àquele, o impacto do subsídio no preço mundial poderá ser superior ao limite crítico.

Nas Figuras 1 a 5 observam-se, de maneira geral, os menores níveis nos preços mundiais do período entre os anos de 1999 a 2002. Nesse mesmo período, também ocorreram os maiores níveis de subsídios domésticos americanos.

Considerando os valores dos parâmetros descritos na Tabela 2 e os níveis de subsídios das caixas amarela e azul descritos anteriormente, a Tabela 2 mostra os valores dos níveis de distorção nos preços mundiais dos produtos analisados neste estudo. Os resultados descritos nesta tabela mostram um maior nível de distorção no preço mundial naqueles anos de menores preços mundiais ${ }^{3}$. Tais valores corroboram os níveis de impacto nos preços mundiais descritos por Sumner (2005), e pequenas diferenças nestes valores ocorrem em função das diferenças nos valores das elasticidades utilizadas.

Este estudo utilizou um cenário de preço médio em três anos consecutivos de baixos níveis de preços mundiais, levando em conta os preços do período

\footnotetext{
3 Descrição metodológica utilizada para as estimativas realizadas na Figura 6 encontra-se em Sumner (2005).
} 


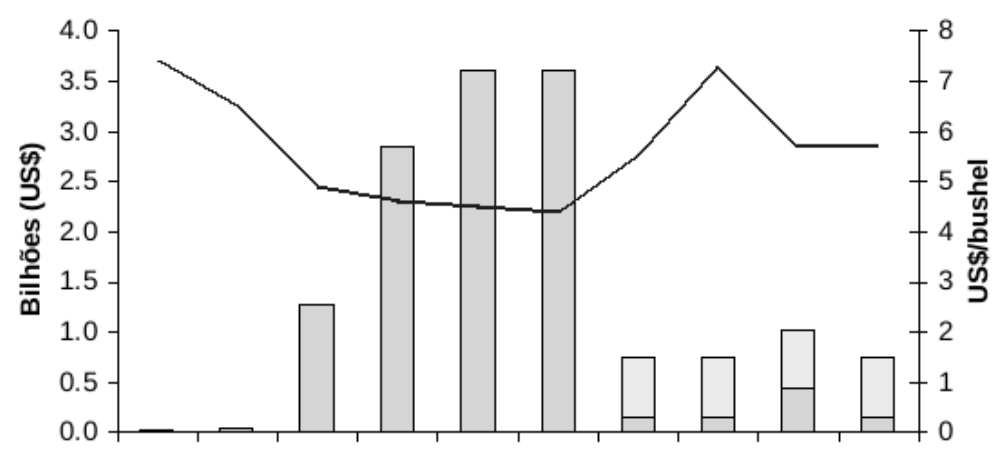

96/97 97/98 98/99 99/00 00/01 01/02 02/03 03/04 04/05 05/06

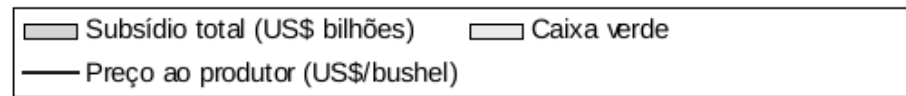

Fonte: USDA (2007); notificações da OMC (2007); Simulações ICONE.

Figura 1: Preço de soja no mercado americano e nível de subsídios dados para a soja (caixas amarela e azul em laranja e caixa verde em verde) em anos recentes
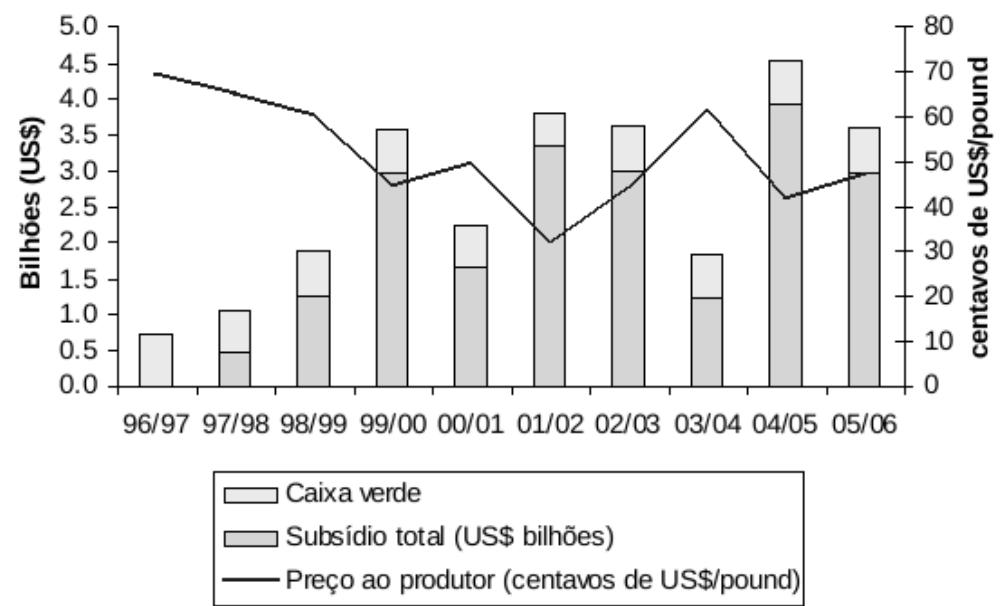

Fonte: USDA (2007); notificações da OMC (2007); Simulações ICONE.

Figura 2: Preço de algodão no mercado americano e nível de subsídios dados para o algodão (caixas amarela e azul em laranja e caixa verde em verde) em anos recentes 

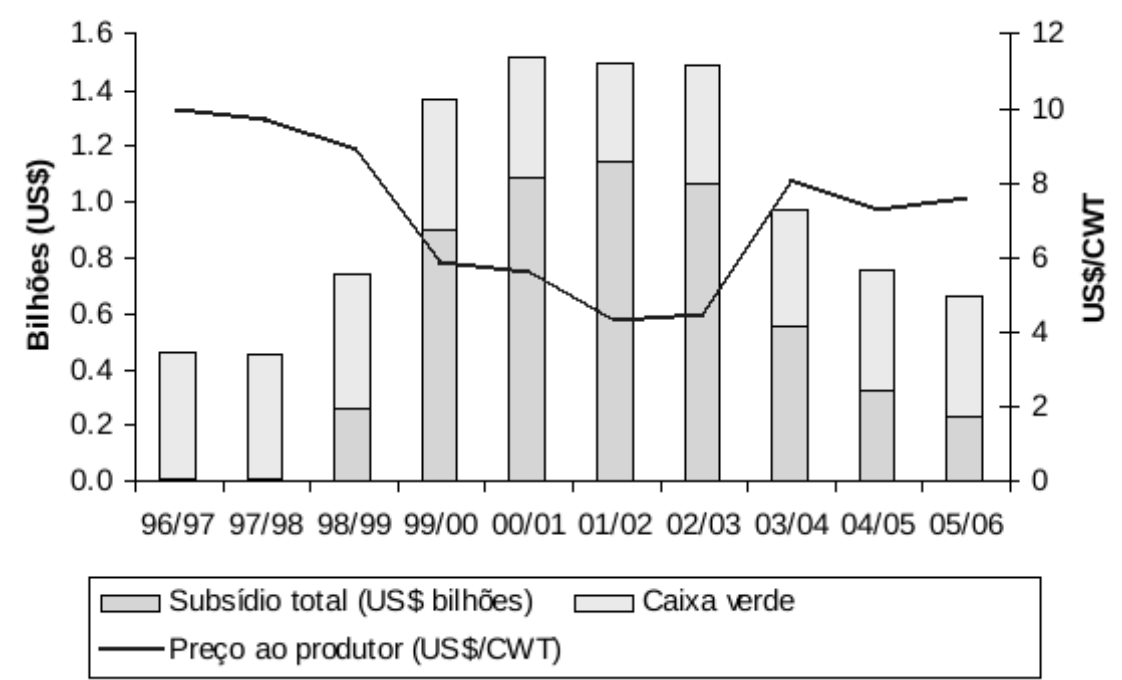

Fonte: USDA (2007); notificações da OMC (2007); Simulações ICONE.

Figura 3: Preço de arroz no mercado americano e nível de subsídios dados para o arroz (caixas amarela e azul em laranja e caixa verde em verde) em anos recentes

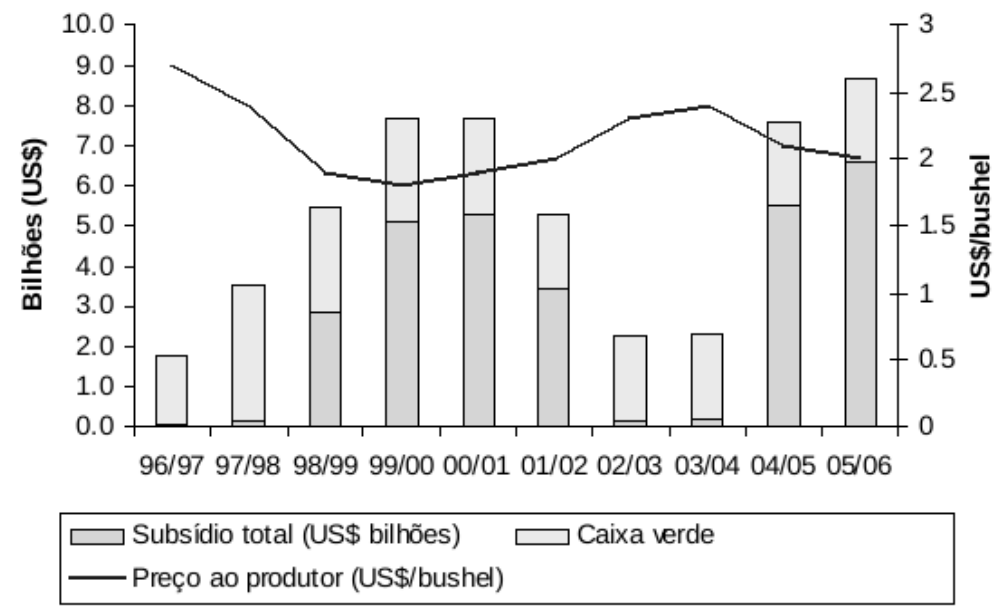

Fonte: USDA (2007); notificações da OMC (2007); Simulações ICONE.

Figura 4: Preço de milho no mercado americano e nível de subsídios dados para o milho (caixas amarela e azul em laranja e caixa verde em verde) em anos recentes 

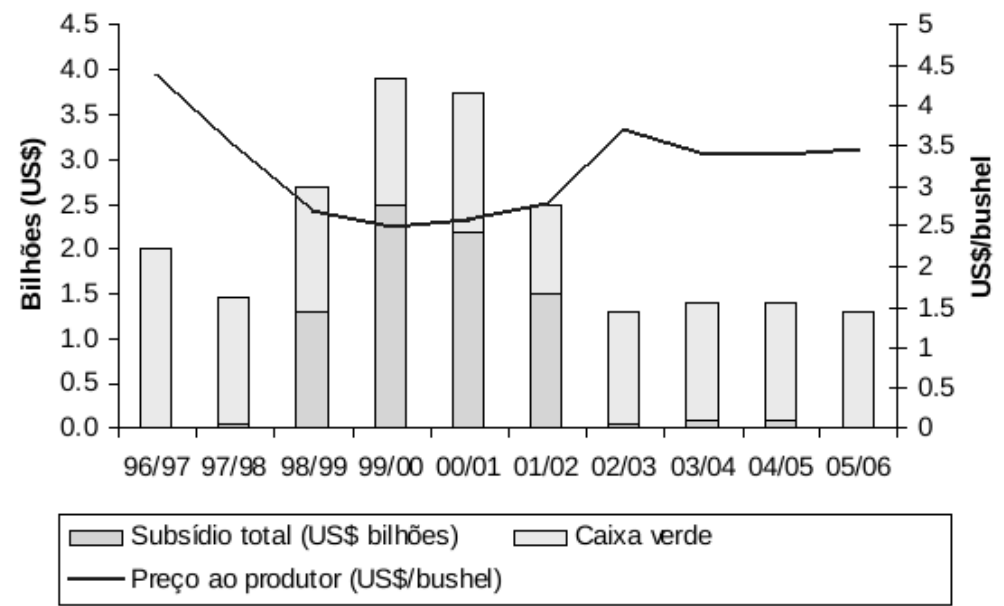

Fonte: USDA (2007); notificações da OMC (2007); Simulações ICONE.

Figura 5: Preço de trigo no mercado americano e nível de subsídios dados para o trigo (caixas amarela e azul em laranja e caixa verde em verde) em anos recentes

Tabela 3: Efeitos adversos no preço mundial de soja, algodão, arroz, milho e trigo considerando os níveis dos subsídios americanos distorcivos em vários anos

\begin{tabular}{lrrrrrrrrrr}
\hline & $97 / 98$ & $98 / 99$ & $99 / 00$ & $00 / 01$ & $01 / 02$ & $02 / 03$ & $03 / 04$ & $04 / 05$ & $05 / 06$ \\
\hline Soja & $-0,10$ & $-2,40$ & $-5,60$ & $-6,80$ & $-6,50$ & $-0,20$ & $-0,20$ & $-0,60$ & $-0,20$ \\
Algodão & $-1,00$ & $-2,60$ & $-6,50$ & $-3,80$ & $-8,20$ & $-6,90$ & $-2,50$ & $-6,70$ & $-5,10$ \\
Arroz & 0,00 & $-0,90$ & $-3,30$ & $-4,60$ & $-4,40$ & $-5,00$ & $-2,30$ & $-1,10$ & $-0,90$ \\
Milho & $-0,20$ & $-3,70$ & $-6,80$ & $-7,00$ & $-4,30$ & $-0,20$ & $-0,20$ & $-5,50$ & $-6,90$ \\
Trigo & $-0,10$ & $-2,00$ & $-3,80$ & $-3,40$ & $-2,10$ & 0,00 & $-0,20$ & $-0,10$ & 0,00 \\
\hline
\end{tabular}

Fonte: Cálculos dos autores.

Tabela 4: Preços e quantidades utilizados no cálculo da equação (9)

\begin{tabular}{lcclrl}
\hline & Período & & Preço médio & \multicolumn{2}{c}{ Quantidade média } \\
\hline Soja & $1999-2001$ & 4,52 & US\$ per Bushel & 2.653 .733 .098 & Bushel \\
Algodão & $2000-2002$ & 935 & US\$ per Ton & 3.969 .881 & Ton \\
Arroz & $2000-2002$ & 4,78 & US\$ per Hundredweight & 127.940 .000 & cwt \\
Milho & $1998-2000$ & 1,87 & US\$ per Bushel & 9.701 .444 .551 & Bushel \\
Trigo & $1998-2000$ & 2,58 & US\$ per Bushel & 2.356 .983 .637 & Bushel \\
\hline
\end{tabular}

Fonte: USDA (2007), FAPRI (2007).

de 1995 a 2005. Isso ocorreu, em geral, entre 1999 e 2002. Nesse período, as commodities analisadas apresentaram os maiores níveis de distorção nos preços mundiais, como descrito na Tabela 2.

O nível de produção utilizado para calcular a equação (11) foi a média para o mesmo período do preço selecionado. A Tabela 4 descreve os valores de preço e quantidade utilizados no estudo.

Utilizando as informações calculadas, pesquisadas e analisadas anteriormente, foram descritos os valores de subsídio nos EUA que causam o nível de dano estabelecido como teto sobre o preço mundial para os produtos se- 
Tabela 5: Resultados do valor do subsídio estimados que distorce $2 \%$ e $4 \%$ do preço mundial da soja e $10 \%$ do valor da produção (média 1999-2005), em bilhões de dólares

\begin{tabular}{lccc}
\hline & \multicolumn{2}{c}{$\begin{array}{c}\text { Distorção no } \\
\text { preço mundial }\end{array}$} & $\begin{array}{c}\text { Distorção no valor } \\
\text { da produção (média } \\
1999 \text { a 2005) } \\
\end{array}$ \\
$2 \%$ & $4 \%$ & $10 \%$ \\
\hline Soja & 1,01 & 2,10 & 1,52 \\
Algodão & 0,60 & 1,36 & 0,51 \\
Arroz & 0,20 & 0,48 & 0,13 \\
Milho & 1,40 & 2,92 & 2,08 \\
Trigo & 1,35 & 3,09 & 0,64 \\
\hline Fonte: elaboraça
\end{tabular}

Fonte: elaboração dos autores.

lecionados. Com base nesses resultados, foi também descrita uma proposta de disciplina por produto com o objetivo de ser negociada entre os países na Rodada de Doha.

\subsection{Limites dos subsídios e propostas de corte para as negociações agrícolas}

Os resultados apresentados na Tabela 5 foram obtidos considerando o cenário de preço e nível de produção descritos na Tabela 4. Tais resultados mostram o valor dos subsídios agrícolas dados aos produtores americanos e qual deveria ser o nível daquele subsídio de maneira a provocar uma distorção de $2 \%$ e $4 \%$ naqueles preços, considerando o dado médio de um período de três anos de baixo nível do preço mundial, entre 1999 e 2002. O nível de 4\% de distorção foi utilizado apenas como uma comparação dos valores, uma vez que, como mencionado, já é um nível alto de distorção que um subsídio poderia produzir.

Na Tabela 5 foram descritos também o valor de $10 \%$ no nível de produção (média para os anos de 1999 a 2005). Verifica-se que o nível de subsídio que pode distorcer o preço mundial em cerca de $2 \%$ é, em geral, próximo àqueles valores. Isso é importante para se sugerir uma proposta de disciplina por produto para apoio doméstico, aplicado pelos países desenvolvidos, que seja de fácil mensuração e aplicação.

De maneira geral, comparando os valores dos subsídios que os EUA aplicaram nos anos de maiores preços de mercado (Figuras 1 a 5), observa-se que eles coincidem com um impacto no preço mundial em cerca dos $2 \%$. E aqueles subsídios se apresentaram muito inferiores aos observados em condições de preços menores. Isso é mais uma constatação do percentual de $2 \%$ de impacto no preço mundial como um nível tolerável para se buscar nas negociações de disciplinas por produto.

Na soja, verifica-se que $10 \%$ do valor médio da produção americana foi equivalente a 1,5 bilhão de dólares, nível ainda acima daquele que foi calculado para distorcer $2 \%$ do preço mundial (1,01 bilhão de dólares). Esse nível foi semelhante àquele utilizado no mercado norte-americano em 1998, quando os preços mundiais da soja estavam elevados. Considerando os maiores níveis aplicados no período (US\$ 3,6 bilhões, em 2001) esse valor repre- 
senta um corte aplicado máximo de $61 \%$ no maior nível de subsídio.

No mercado de algodão, os níveis de subsídio que distorcem $2 \%$ do preço mundial no cenário de preços baixos (US\$ 0,60 bilhão) são coincidentes com $10 \%$ do valor médio da produção (US\$ 0,51 bilhão). O nível de corte efetivo neste caso é superior aos demais: $84 \%$ dos subsídios aplicados no ano de menor preço (2001). A necessidade desse corte demonstra o quão distorcivo se apresentou aquele nível de subsídio.

Resultado semelhante ao descrito anteriormente para o mercado do algodão foi obtido para o arroz. Assim, o nível de $10 \%$ do valor médio da produção norte-americana (US\$ 0,13 bilhão) foi também semelhante ao nível de subsídio capaz de distorcer o preço mundial em $2 \%$ num cenário de preços baixos (US\$ 0,20 bilhão). Tais níveis foram bastante inferiores ao subsídio oferecido no ano de maior preço (US\$ 0,97 bilhão). Assim, o corte efetivo foi de $90 \%$ dos subsídios aplicados no ano de menor preço (2001). Deve-se lembrar que, para este mercado, foram utilizadas as participações norte-americanas no mercado internacional e não no mercado total. Entretanto, isso foi justificado pelo alto nível de produção do produto para subsistência no mercado mundial.

Para o mercado norte-americano de milho, o valor de $10 \%$ do valor médio da produção (US\$2,08 bilhões) representa um corte efetivo no maior nível aplicado no período (US\$7,6 bilhões, em 2001), de 74\%. O nível de subsídio que poderia distorcer $2 \%$ do preço mundial da commodity foi calculado em US\$ 1,4 bilhão. Da mesma forma que nos mercados descritos anteriormente, $10 \%$ do valor de produção coincidiu com aquele nível de subsídio.

Finalmente, o mercado americano de trigo apresentou um limite de subsídio máximo que distorce o preço mundial dentro do limite tolerável estabelecido de US\$1,35 bilhão, e $10 \%$ do valor médio da produção foi calculado em US\$ 0,64 bilhão. O corte efetivo considerando o subsídio aplicado no ano de menor preço (1999) e o valor de $10 \%$ do valor da produção corresponde a $79 \%$.

Verifica-se, portanto, nos casos analisados, que o indicador de $10 \%$ do valor da produção apresenta-se como um bom indicativo como proposta para aplicação de disciplina por produto a ser negociada na Rodada de Doha.

\section{Conclusão}

Os subsídios domésticos dados pelos países desenvolvidos aos produtos agrícolas vêm sendo contestados em painéis na OMC como causadores de distorções no mercado mundial. Esses contenciosos vêm dando ganhos de causa aos países contestadores. Por essa razão, novos processos de solução de controvérsias têm sido abertos a pedido de países interessados. Entretanto, os painéis são dispendiosos e os países requerentes são, em geral, em desenvolvimento, exportadores de commodities agrícolas.

O Programa de Trabalho (Framework) da Rodada de Doha, de agosto de 2004, estabelece: "The Doha Ministerial Declaration calls for 'substantial reductions in trade-distorting domestic support." Portanto, a identificação dos subsídios que distorcem o mercado mundial é essencial para se saber qual limite deve ser estabelecido nestas rodadas de negociações multilaterais. Tal objetivo vem ao encontro dos objetivos dos contenciosos realizados no âmbito da OMC. Assim, o presente trabalho buscou, levando em consideração o que foi realizado nos contenciosos, estabelecer um padrão para limites nos subsídios 
atualmente aplicados. Tais limites devem suprir, por sua vez, as exigências estabelecidas no parágrafo terceiro do Artigo 6 do Acordo Sobre Subsídios e Medidas Compensatórias. Isso eliminaria a necessidade de outros painéis para os produtos agrícolas na OMC.

Entretanto, as negociações da OMC são realizadas para todos os produtos e países, não levando em consideração, de maneira geral, casos específicos a serem atribuídos a países ou produtos. Assim, a metodologia utilizada para estimar o limite de subsídio que impacta em $2 \%$ o preço mundial do produto poderia não ser viável, pela sua complexidade nos cálculos e necessidade de parâmetros estimáveis. Nesse sentido, procurou-se encontrar paralelismo entre os resultados obtidos com possíveis propostas factíveis de uso nas negociações. Tal paralelismo pode ser obtido considerando o limite de $10 \%$ do valor da produção para um período determinado (média, ano 1999-2005). Nesse sentido, este estudo busca ter um caráter prescritivo para estabelecer limites de apoio doméstico nas disciplinas por produto a serem negociados na Rodada de Doha.

\section{Referências Bibliográficas}

A. S. P. Brandão e E. C. R. Lima. Impacts of the us subsidy to soybeans on world prices, production and exports. Revista de Economia e Sociologia Rural, 44:631-676, 2006.

M. Jales e A. M. Nassar. How to read the us and eu proposals on domestic support to agriculture. Weekly Trade News Digest, 10, 2005.

D. Poonyth, A. Sarris, R. Sharma, e S. Shui. The impact of domestics and trade policies on the world cotton market. Technical report, 2004.

D. A. Sumner. A quantitative simulation analysis of the impacts of u.s. cotton subsidies on cotton prices and quantities. Mimeo, 2003.

D. A. Sumner. Conflicts between u.s. farm policies and wto obligations. Technical report, Trade Policy Analysis, 32, december 2005.

WTO.http://www.wto.org/english/thewto_e/countries_e/usa_e.htm.agriculture.2007, 2007. URL http://www.wto.org/english/thewto_e/countries_e/usa_e.htm. agriculture.2007. 